Mon. Not. R. Astron. Soc. 000,19 (2021) Printed 16 October $2020 \quad$ (MN LATEX style file v2.2)

\title{
From lenticulars to blue compact dwarfs: the stellar mass fraction is regulated by disc gravitational instability
}

\author{
Alessandro B. Romeo, ${ }^{1 \star}$ Oscar Agertz ${ }^{2}$ and Florent Renaud ${ }^{2}$ \\ ${ }^{1}$ Department of Space, Earth and Environment, Chalmers University of Technology, SE-41296 Gothenburg, Sweden \\ ${ }^{2}$ Department of Astronomy and Theoretical Physics, Lund University, Box 43, SE-22100 Lund, Sweden
}

Accepted 2020 October 15. Received 2020 October 14; in original form 2020 June 16

\begin{abstract}
The stellar-to-halo mass relation (SHMR) is not only one of the main sources of information we have on the connection between galaxies and their dark matter haloes, but also an important indicator of the performance of galaxy formation models. Here we use one of the largest sample of galaxies with both high-quality rotation curves and near-infrared surface photometry, and perform a detailed comparative analysis of the SHMR. Our analysis shows that there are significant statistical differences between popular forms of the SHMR, and illustrates the predictive power of a new physically motivated scaling relation, which connects the stellar mass fraction $\left(M_{\star} / M_{\mathrm{h}}\right)$ to the stellar specific angular momentum $\left(j_{\star}\right)$ and the stellar radial velocity dispersion $\left(\sigma_{\star}\right)$ via disc gravitational instability. Making use of such a relation, we demonstrate (i) how challenging it is to reproduce the efficiency of galaxy formation even for stateof-the-art cosmological hydrodynamical simulations, and (ii) that the evolution of the stellar mass fraction is regulated by disc gravitational instability: when $M_{\star} / M_{\mathrm{h}}$ varies, $j_{\star}$ and $\sigma_{\star}$ also vary as predicted by our scaling relation, thus erasing the memory of such evolution. This implies that the process of disc gravitational instability is intriguingly uniform across disc galaxies of all morphological types: from lenticulars to blue compact dwarfs. In particular, the cosmic variance of Toomre's $Q$ is 0.2 dex, a universal value for both stars and atomic gas.
\end{abstract}

Key words: instabilities - galaxies: fundamental parameters - galaxies: haloes galaxies: kinematics and dynamics - galaxies: stellar content - dark matter.

\section{INTRODUCTION}

Galaxy formation is an elaborate process involving mechanisms such as the accretion of baryons onto dark matter haloes, galaxy mergers, cooling and heating, gravitational instabilities, star formation, and feedback from massive stars. Despite this complexity, galaxies tend to be well described by a set of scaling relations. These are simple power laws that relate galaxy structural parameters such as size, luminosity, rotational velocity and angular momentum (see, e.g., Cimatti et al. 2020).

A fundamental aspect of galaxy formation is the connection between galaxy and host dark matter halo properties (see, e.g., Wechsler \& Tinker 2018). A number of different methods are used to characterize the stellar-to-halo mass relation (SHMR), including halo abundance matching (e.g., Vale \& Ostriker 2004; Conroy et al. 2006; Moster et al. 2010; Behroozi et al. 2019), satellite kinematics (e.g., Klypin \& Prada 2009; More et al. 2011), weak lensing (e.g., Man-

* E-mail: romeo@chalmers.se delbaum et al. 2006; Leauthaud et al. 2012), and internal galaxy dynamics (e.g., Read et al. 2017). These techniques all independently point to galaxy formation being a highly inefficient process, with stars making up at most a few per cent of the halo mass in Milky Way-like systems, and significantly less in dwarf galaxies. Understanding the origins of this inefficiency, and of scaling relations in general, is a central theme in galaxy formation.

Today, several decades after the pioneering work of Ostriker \& Peebles (1973) and the seminal papers by Efstathiou et al. (1982), Christodoulou et al. (1995) and Mo et al. (1998), theoretical work is till providing fresh insights into the link between gravitational instability and galaxy evolution, which turn out to be useful for exploring the galaxyhalo connection from an entirely different perspective (e.g., Athanassoula 2008; Agertz \& Kravtsov 2016; Sellwood 2016; Okamura et al. 2018; Romeo \& Mogotsi 2018; Zoldan et al. 2018; Valencia-Enríquez et al. 2019; Romeo 2020; Zanisi et al. 2020).

Romeo \& Mogotsi (2018) analysed a galaxy property that is not directly observable but is tightly constrained by 
gravitational instability and other fundamental physical processes: $\left\langle\mathcal{Q}_{\star}\right\rangle$, the mass-weighted average of the stellar $Q$ stability parameter (Toomre 1964), corrected so as to include disc thickness effects (Romeo \& Falstad 2013). They showed that $\left\langle\mathcal{Q}_{\star}\right\rangle$ is remarkably constant across spiral galaxies of all morphological types (Sa-Sd), $\left\langle\mathcal{Q}_{\star}\right\rangle \approx 2-3$, thus proving the existence of the self-regulation process predicted by pioneering simulation work on spiral structure (e.g., Miller et al. 1970; Hohl 1971; Sellwood \& Carlberg 1984; Carlberg \& Sellwood 1985) 1

Romeo (2020) extended the analysis carried out by Romeo \& Mogotsi (2018) to molecular and atomic gas, and provided tighter constraints on how self-regulated galaxy discs are. He showed that the median value of $\langle Q\rangle$ across the $\mathrm{Sa}-\mathrm{Sd}$ sequence varies significantly not only from stars to gas but also between the molecular and atomic gas phases, while the $1 \sigma$ scatter of $\langle Q\rangle$ is small for all the components. Romeo (2020) further demonstrated that the low galaxy-togalaxy variance of $Q$ translates into a simple formula, which has important astrophysical applications: it allows one to generate new physically motivated galaxy scaling relations, all driven by disc gravitational instability. An eloquent example is provided by the atomic gas-to-stellar mass relation (see figs 4 and 5 of Romeo 2020).

In this paper, we generate one such relation and test it against two popular forms of the SHMR. While the present analysis builds on the approach developed by Romeo (2020), the application presented here is new and stretches across galaxies spanning a wide range of morphological types (S0BCD), stellar masses $\left(M_{\star} \approx 10^{6.5-11.5} \mathrm{M}_{\odot}\right)$ and halo masses $\left(M_{\mathrm{h}} \approx 10^{9-13} \mathrm{M}_{\odot}\right)$. The rest of the paper is organized as follows. In Sect. 2, we describe the galaxy sample, data and statistics. In Sect. 3, after summarizing why such a galaxy sample is especially appropriate for analysing the SHMR, we perform a detailed comparative analysis of the popular $M_{\star} / M_{\mathrm{h}}$ vs $M_{\mathrm{h}}$ and $M_{\star} / M_{\mathrm{h}}$ vs $M_{\star}$ as well as of our scaling relation. In Sect. 4 , we demonstrate the importance of our results for the simulation community, especially in the context of the ongoing effort to reproduce the efficiency of galaxy formation in cosmological hydrodynamical simulations. Finally, in Sect. 5, we draw the conclusions of our work and discuss its astrophysical implication.

\section{GALAXY SAMPLE, DATA AND STATISTICS}

To analyse the SHMR, we use a sample of 142 galaxies, ranging from lenticulars to blue compact dwarfs, and spanning four and a half orders of magnitude in $M_{\star}$ and $M_{\mathrm{h}}$. Our galaxy sample is a combination of the samples analysed by Posti et al. (2018, 2019; hereafter P18, P19) and Iorio et al. (2017), which were selected from two databases: Spitzer Photometry and Accurate Rotation Curves (SPARC; Lelli et al. 2016) and Local Irregulars That Trace Luminosity Extremes, The H i Nearby Galaxy Survey (LITTLE THINGS; Hunter et al. 2012), respectively. Like those samples, our

\footnotetext{
1 Romeo \& Mogotsi (2018) also showed that $\left\langle\mathcal{Q}_{\star}\right\rangle$ is related to the Efstathiou, Lake \& Negroponte (1982) global stability parameter, $\epsilon_{\mathrm{m}}$, via the degree of rotational support, $V / \sigma$, and the velocity dispersion anisotropy, $\sigma_{z} / \sigma_{R}$. These are two important effects that are missing from $\epsilon_{\mathrm{m}}$.
}

galaxy sample is neither statistically complete nor volumelimited, but it is nevertheless representative of the full population of (regularly rotating) nearby late-type galaxies (SPARC), with an emphasis on the faint end of the luminosity function (LITTLE THINGS). To the best of our knowledge, this is one of the largest sample of galaxies with both high-quality rotation curves and near-infrared surface photometry (see references above).

Our analysis requires three key quantities: $M_{\star}, M_{\mathrm{h}}$ and $j_{\star} \equiv J_{\star} / M_{\star}$, the stellar specific angular momentum. As regards the accuracy of such measurements, we distinguish four galaxy subsamples, which we name and describe below.

(i) 'SPARC +++ ' contains $75 \mathrm{~S} 0-\mathrm{BCD}$ galaxies with accurate measurements of $M_{\star}, M_{\mathrm{h}}$ and $j_{\star}$. The stellar and halo masses were measured by P19 via rotation curve decomposition, adopting a standard NFW halo model (Navarro et al. 1996) and a Bayesian approach to fit the observed rotation curves. The stellar specific angular momentum was measured by $\mathrm{P} 18$ via radial integration, imposing a convergence criterion on the cumulative $j_{\star}(<R)$ profile and including asymmetric drift corrections (see, e.g., Binney \& Tremaine 2008, chap. 4.8.2).

(ii) 'SPARC++' contains $35 \mathrm{~S}-\mathrm{BCD}$ galaxies with accurate measurements of $M_{\star}$ and $M_{\mathrm{h}}$, and approximate estimates of $j_{\star}$. The stellar and halo masses were measured by $\mathrm{P} 19$, as in sample (i). For these galaxies the cumulative $j_{\star}(<R)$ profile does not converge, hence the radial integration described in P18 would only yield a lower limit on $j_{\star}$. For this reason, we estimate the stellar specific angular momentum adopting a commonly used approximation, $j_{\star}=2 R_{\mathrm{d}} V_{\text {flat }}$, where $R_{\mathrm{d}}$ is the exponential disc scale length and $V_{\text {flat }}$ is the velocity along the flat part of the rotation curve (e.g., Romanowsky \& Fall 2012). We take $R_{\mathrm{d}}$ and $V_{\text {flat }}$ from Lelli et al. (2016).

(iii) 'SPARC+' contains $15 \mathrm{~S}-\mathrm{Im}$ galaxies with accurate measurements of $j_{\star}$, and approximate estimates of $M_{\star}$ and $M_{\mathrm{h}}$. The stellar specific angular momentum was measured by $\mathrm{P} 18$, as in sample (i). For these galaxies the rotation curve decomposition described in P19 does not yield a unimodal posterior distribution for $M_{\mathrm{h}}$. This happens for various reasons, so Lorenzo Posti adopted various strategies to estimate the halo mass and kindly provided us with such estimates. Nine galaxies (mostly dwarfs) have slowly rising rotation curves, so a cored halo model (Burkert 1995) was used to get an adequate fit. Three other galaxies are nearly edge-on spirals for which the innermost points of the rotation curve are very uncertain, so the fit was repeated excluding those points. The last three galaxies of the sample are spirals that have quite noisy and poorly sampled rotation curves, which give rise to secondary peaks in the posterior distribution of $M_{\mathrm{h}}$; in such a case, only the main peak of the posterior was considered.

(iv) 'LITTLE THINGS' contains 17 dwarf irregular (Im) galaxies with reliable measurements of $M_{\star}$ and $M_{\mathrm{h}}$, and approximate estimates of $j_{\star}$. The stellar and halo masses were measured by Read et al. (2017) via rotation curve decomposition. We estimate the stellar specific angular momentum as in sample (ii), i.e. as $j_{\star}=2 R_{\mathrm{d}} V_{\text {flat }}$. We take $R_{\mathrm{d}}$ from Hunter \& Elmegreen (2006), and $V_{\text {flat }}$ from Iorio et al. (2017).

In addition to the key quantities specified above $\left(M_{\star}\right.$, $M_{\mathrm{h}}$ and $j_{\star}$ ), we need to quantify the morphological type 

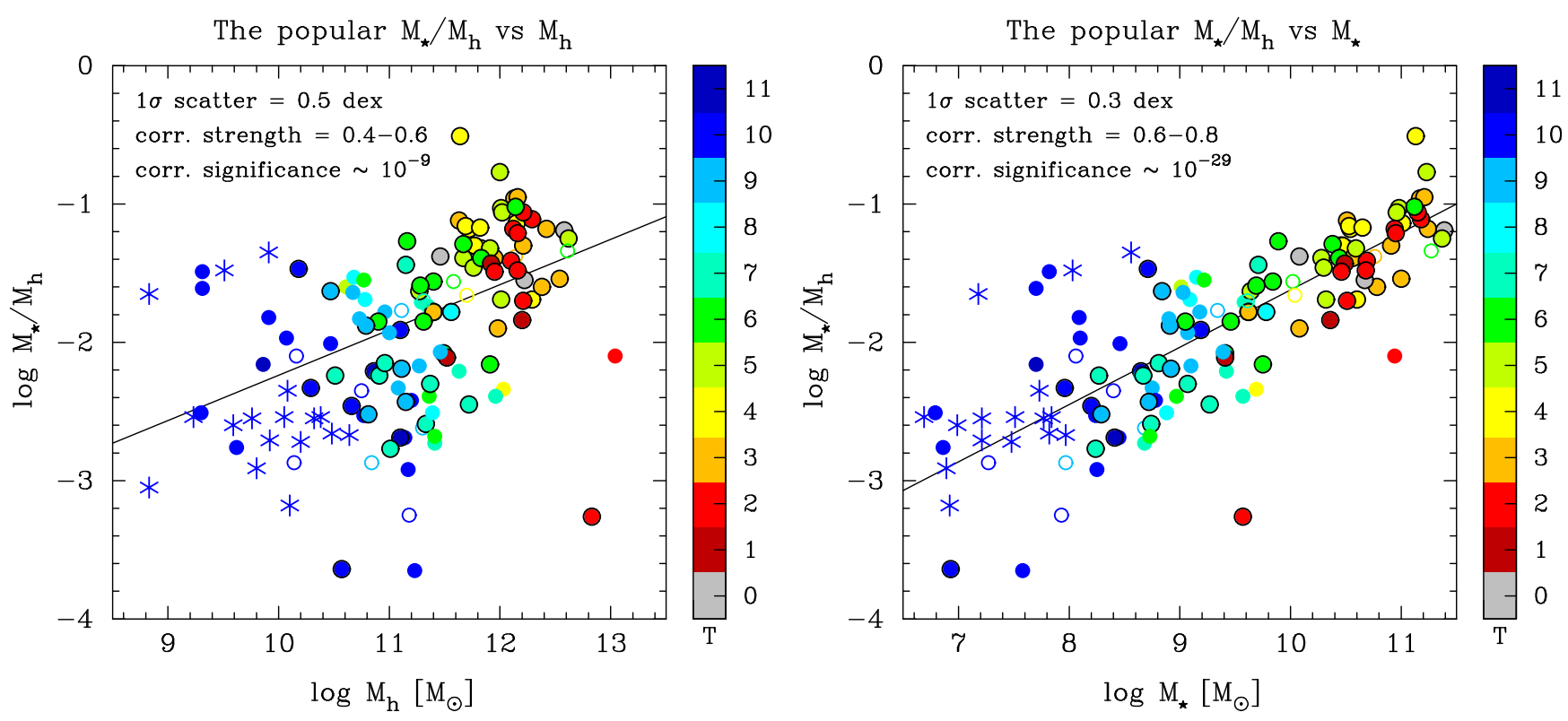

Figure 1. Comparison between two popular forms of the SHMR. Galaxies are colour-coded by Hubble stage, and galaxy samples are symbol-coded by the accuracy of the $M_{\star}, M_{\mathrm{h}}$ and $j_{\star}$ measurements (see Sect. 2 for more information): SPARC +++ (solid circles with black ouline), SPARC ++ (solid circles), SPARC + (hollow circles), LITTLE THINGS (asterisks). The lines in the left and right panels, $\log \left(M_{\star} / M_{\mathrm{h}}\right)=0.33 \log M_{\mathrm{h}}-5.51$ and $\log \left(M_{\star} / M_{\mathrm{h}}\right)=0.41 \log M_{\star}-5.76$, are robust median-based fits to the data points. Statistical information about the data is given in summary form and simplified notation (see Sect. 2 for more information).

and the gas mass of each galaxy. The morphological type is taken from Lelli et al. (2016) for SPARC galaxies, and from Hunter et al. (2012) for LITTLE THINGS galaxies. The gas mass is computed as $M_{\text {gas }}=1.33 M_{\mathrm{HI}}$, i.e. neglecting the contribution of molecular gas (CO data are not available for most galaxies of our sample) but including the contribution of helium to the atomic gas mass. $M_{\mathrm{HI}}$ is taken from Lelli et al. (2016) for SPARC galaxies, and from Iorio et al. (2017) for LITTLE THINGS galaxies.

Finally, to quantify the tightness, correlation strength and significance of the SHMR, we present the results of several statistical measures and associated tests. First of all, we measure the dispersion of the data points around the model using a robust estimator of the $1 \sigma$ scatter: $\mathrm{SD}_{\text {rob }}=$ $1 / 0.6745 \mathrm{MAD}$, where $\mathrm{SD}_{\text {rob }}$ is the robust counterpart of the standard deviation and MAD is the median absolute deviation (see, e.g., Müller 2000). Values of $\mathrm{SD}_{\text {rob }}$ that are much less than the dynamic range of the data mean a tight relation. Secondly, we measure Pearson's $r$, Spearman's $\rho$ and Kendall's $\tau$ correlation coefficients, together with their significance levels $p_{r}, p_{\rho}$ and $p_{\tau}$ (see, e.g., Press et al. 1992, chaps 14.5 and 14.6). Values of $r, \rho, \tau \approx(-) 1$ and $p_{r}, p_{\rho}, p_{\tau} \approx$ 0 mean a strong and significant (anti)correlation. In Sect. 3, we will provide all such statistical information in summary form and simplified notation.

\section{COMPARATIVE ANALYSIS OF THE SHMR}

The galaxy sample described in Sect. 2 is especially appropriate for analysing the SHMR. SPARC is one of the largest collection of galaxies with both high-quality rotation curves and near-infrared surface photometry (Lelli et al. 2016). This allows measuring $M_{\star}$ and $M_{\mathrm{h}}$ accurately via rotation curve decomposition (P19). The LITTLE THINGS galaxies included in the sample also have reliable measurements of $M_{\star}$ and $M_{\mathrm{h}}$, again derived via rotation curve decomposition (Iorio et al. 2017; Read et al. 2017). So, although our sample does not contain elliptical galaxies by construction, it stretches across all other morphological types (S0-BCD) with high data quality. This allows us to perform a detailed comparative analysis of the SHMR, and thus to probe the similarities and the differences between two popular forms of the SHMR and our scaling relation. This section presents the results of such an analysis.

\subsection{Two popular forms of the SHMR}

The SHMR has been massively studied adopting two alternative parametrizations: $M_{\star} / M_{\mathrm{h}}$ vs $M_{\mathrm{h}}$ (e.g., Leauthaud et al. 2012; Behroozi et al. 2013; Moster et al. 2013; RodríguezPuebla et al. 2015; van Uitert et al. 2016; Girelli et al. 2020), and/or $M_{\star} / M_{\mathrm{h}}$ vs $M_{\star}$ (e.g., Dutton et al. 2010; Leauthaud et al. 2012; van Uitert et al. 2016; Lapi et al. 2018; P19). To the best of our knowledge, these two relations have not yet been compared using data of such high quality as those described in Sect. 2. Here we perform such a comparison.

Fig. 1 and the statistical information shown in the two panels illustrate that the two popular forms of the SHMR are not at all equivalent. $M_{\star} / M_{\mathrm{h}}$ vs $M_{\mathrm{h}}$ is less constrained than $M_{\star} / M_{\mathrm{h}}$ vs $M_{\star}$, as indicated for instance by its larger $1 \sigma$ scatter. This is true whether we consider the whole galaxy sample or SPARC +++ , i.e. the galaxies with most accurate measurements of $M_{\star}$ and $M_{\mathrm{h}}$ (as well as $j_{\star}$ ). Indeed, $M_{\star} / M_{\mathrm{h}}$ vs $M_{\mathrm{h}}$ scatters less in SPARC $+++\left(\mathrm{SD}_{\text {rob }} \approx 0.4 \mathrm{dex}\right)$ than in the whole sample $\left(\mathrm{SD}_{\text {rob }} \approx 0.5 \mathrm{dex}\right)$, but not as little as $M_{\star} / M_{\mathrm{h}}$ vs $M_{\star}\left(\mathrm{SD}_{\text {rob }} \approx 0.3 \mathrm{dex}\right)$.

To understand why $M_{\star} / M_{\mathrm{h}}$ vs $M_{\mathrm{h}}$ is poorly constrained 
and learn how to generate an SHMR that is tighter than $M_{\star} / M_{\mathrm{h}}$ vs $M_{\star}$, we need to look at the SHMR from a different perspective. We will do this in Sects 3.2 and 3.3.

\subsection{Disc gravitational instability as a driver of galaxy scaling relations}

As pointed out in Sect. 1, Romeo (2020) demonstrated that disc gravitational instability is a driver of galaxy scaling relations. Here we write down the key equation of that paper, and explain how one can use it for generating new physically motivated scaling relations. In Sect. 3.3, we will illustrate the usefulness of such an approach in the context of the SHMR.

The origin of such relations is the low galaxy-to-galaxy variance of Toomre's (1964) $Q$ stability parameter, which leads to Romeo's (2020) key equation:

$\frac{j_{i} \hat{\sigma}_{i}}{G M_{i}} \approx 1$

Note that this is not a marginal stability condition, but a tight statistical relation between mass $(M)$, specific angular momentum $(j \equiv J / M)$ and velocity dispersion $(\hat{\sigma})$ for each baryonic component in the disc plus bulge: atomic gas $(i=\mathrm{H} \mathrm{I})$, molecular gas $\left(i=\mathrm{H}_{2}\right)$ and stars $(i=\star)$. More precisely, $\hat{\sigma}_{i}$ is the radial velocity dispersion of component $i, \sigma_{i}$, properly averaged and rescaled. This quantity can be evaluated using two alternative equations, depending on whether there are reliable $\sigma_{i}$ measurements available or not. Unfortunately, such measurements are highly non-trivial (e.g., Ianjamasimanana et al. 2017; Marchuk \& Sotnikova 2017), hence very sparse (e.g., Romeo \& Mogotsi 2017; Mogotsi \& Romeo 2019). Therefore, if one wants to analyse a large galaxy sample, then the appropriate equation to use is

$\hat{\sigma}_{i}=\left\{\begin{aligned} 11 \mathrm{~km} \mathrm{~s}^{-1} & \text { if } i=\mathrm{H}_{\mathrm{I}}, \\ 8 \mathrm{~km} \mathrm{~s}^{-1} & \text { if } i=\mathrm{H}_{2}, \\ 130 \mathrm{~km} \mathrm{~s}^{-1} \times\left(M_{\star} / 10^{10.6} \mathrm{M}_{\odot}\right)^{0.5} & \text { if } i=\star .\end{aligned}\right.$

Note that these are not observationally motivated values of the gas and stellar velocity dispersions, but rigorously derived values of the velocity dispersion-based quantity $\hat{\sigma}_{i}$.

To make good use of Eq. (1), one needs to understand three key points:

- Eq. (1) means that $j_{i} \hat{\sigma}_{i} / G$ is an accurate predictor of $M_{i}$. In contrast to other mass estimators, $j_{i} \hat{\sigma}_{i} / G$ is entirely based on the dynamics of galaxy discs, thus it does not rely on any assumption about the CO-to- $\mathrm{H}_{2}$ conversion factor $\left(X_{\mathrm{CO}}\right)$ or the stellar mass-to-light ratio $\left(\Upsilon_{\star}\right)$.

- Eq. (1) can be turned into an equally accurate mass fraction predictor. Simply multiply the left- and right-hand sides by $M_{i} / M_{\text {ref }}$, where $M_{\text {ref }}$ is any well-defined reference mass. By construction, the resulting scaling relation, $M_{i} / M_{\text {ref }}$ vs $G M_{\text {ref }} / j_{i} \hat{\sigma}_{i}$, will have the same (logarithmic) scatter as Eq. (1), which itself is identical to the galaxy-togalaxy scatter of $Q_{i}$. Clearly, the tightness of the resulting scaling relation will depend not only on its scatter, but also on the dynamic range of $M_{i} / M_{\mathrm{ref}}$ : the wider the range, the tighter the relation.

- Eq. (1) applies to spiral galaxies of all morphological types (Sa-Sd). The tests carried out by Romeo (2020) suggest that Eq. (1) has a wider range of applicability (Sa-dIrr), but so far conclusive evidence has only been found for the atomic gas relation (Eq. 1 for $i=\mathrm{H} \mathrm{I}$ ).

\subsection{Our scaling relation, and the roles of specific angular momentum and velocity dispersion}

Now that we know how to make good use of Eq. (1), let us apply such a simple formula in practice. Choosing the mass of the dark matter halo as the reference mass, we get the following predictor for the stellar mass fraction:

$\frac{M_{\star}}{M_{\mathrm{h}}} \approx \frac{j_{\star} \hat{\sigma}_{\star}}{G M_{\mathrm{h}}}$.

Before testing Eq. (3), let us clarify two important points:

- Since $M_{\mathrm{h}}$ plays the role of a reference mass, Eq. (3) contains the same information as the original Eq. (1) $[i=\star]$. This apparently pure stellar equation encloses two layers of information about the dark matter halo. The first layer concerns $j_{\star}$. By definition, $j_{\star}$ is the stellar mass-weighted average of $R V_{\text {rot }}(R)$, the orbital specific angular momentum. Hence $j_{\star}$ depends not only on the mass distribution of the stellar disc, but also on the gravitational potential of the halo. The second layer of information is deeper: it concerns the origin of Eq. (1) $[i=\star]$, which is the remarkably flat radial distribution of $Q_{\star}$ (see fig. 1 of Romeo 2020). The flatness of $Q_{\star}(R)$ indicates that stellar discs self-regulate their mass surface density and radial velocity dispersion so as to lie at the edge of gravitational instability, a state that is dynamically influenced by the gravitational potential of the host dark matter haloes (see again Romeo 2020).

- Since Eq. (3) contains only implicit information about the dark matter halo, $M_{\star} / M_{\mathrm{h}}$ vs $G M_{\mathrm{h}} / j_{\star} \hat{\sigma}_{\star}$ is perhaps not a proper SHMR, but it is nevertheless a physically motivated scaling relation that can provide new insights into the galaxy-halo connection. In fact, $M_{\star} / M_{\mathrm{h}}$ vs $G M_{\mathrm{h}} / j_{\star} \hat{\sigma}_{\star}$ can be directly compared with the popular $M_{\star} / M_{\mathrm{h}}$ vs $M_{\mathrm{h}}$, which is important not only for understanding why $M_{\star} / M_{\mathrm{h}}$ vs $M_{\mathrm{h}}$ is poorly constrained but also for exploring the roles that $j_{\star}$ and $\hat{\sigma}_{\star}$ play in such a scenario.

Fig. 2 shows our scaling relation, $M_{\star} / M_{\mathrm{h}}$ vs $G M_{\mathrm{h}} / j_{\star} \hat{\sigma}_{\star}$, and the impact of suppressing $j_{\star}$ and/or $\hat{\sigma}_{\star}$. Let us first focus on the top-left panel. A comparison with Fig. 1 illustrates that our scaling relation is more tightly constrained than the popular $M_{\star} / M_{\mathrm{h}}$ vs $M_{\mathrm{h}}$ and $M_{\star} / M_{\mathrm{h}}$ vs $M_{\star}$. Indeed, it is striking that a simple formula like Eq. (3), entirely based on a theoretical approach, succeeds in predicting the stellar mass fraction across four orders of magnitude in $M_{\star} / M_{\mathrm{h}}$, to within $0.2 \mathrm{dex}$, and without any free parameter or finetuning. These facts speak clearly. In particular, the small scatter of our scaling relation tells us that the gravitational instability properties of galaxy discs are, on average, remarkably uniform across the sequence S0-BCD. This confirms and extends a result originally found by Romeo (2020) in the context of the atomic gas-to-stellar mass relation. The facts pointed out above are true whether we consider the whole galaxy sample or SPARC +++ . While $M_{\star} / M_{\mathrm{h}}$ vs $M_{\mathrm{h}}$ and $M_{\star} / M_{\mathrm{h}}$ vs $M_{\star}$ scatter less in SPARC +++ than in the whole sample (see Sect. 3.1), our scaling relation is insensitive to the accuracy of the measurements and, whichever (sub)sample is considered, it outperforms the two popular relations in terms of tightness, correlation strength and significance.

There is one more point that we want to understand about our scaling relation. Since it connects three basic quantities (mass, specific angular momentum and velocity 

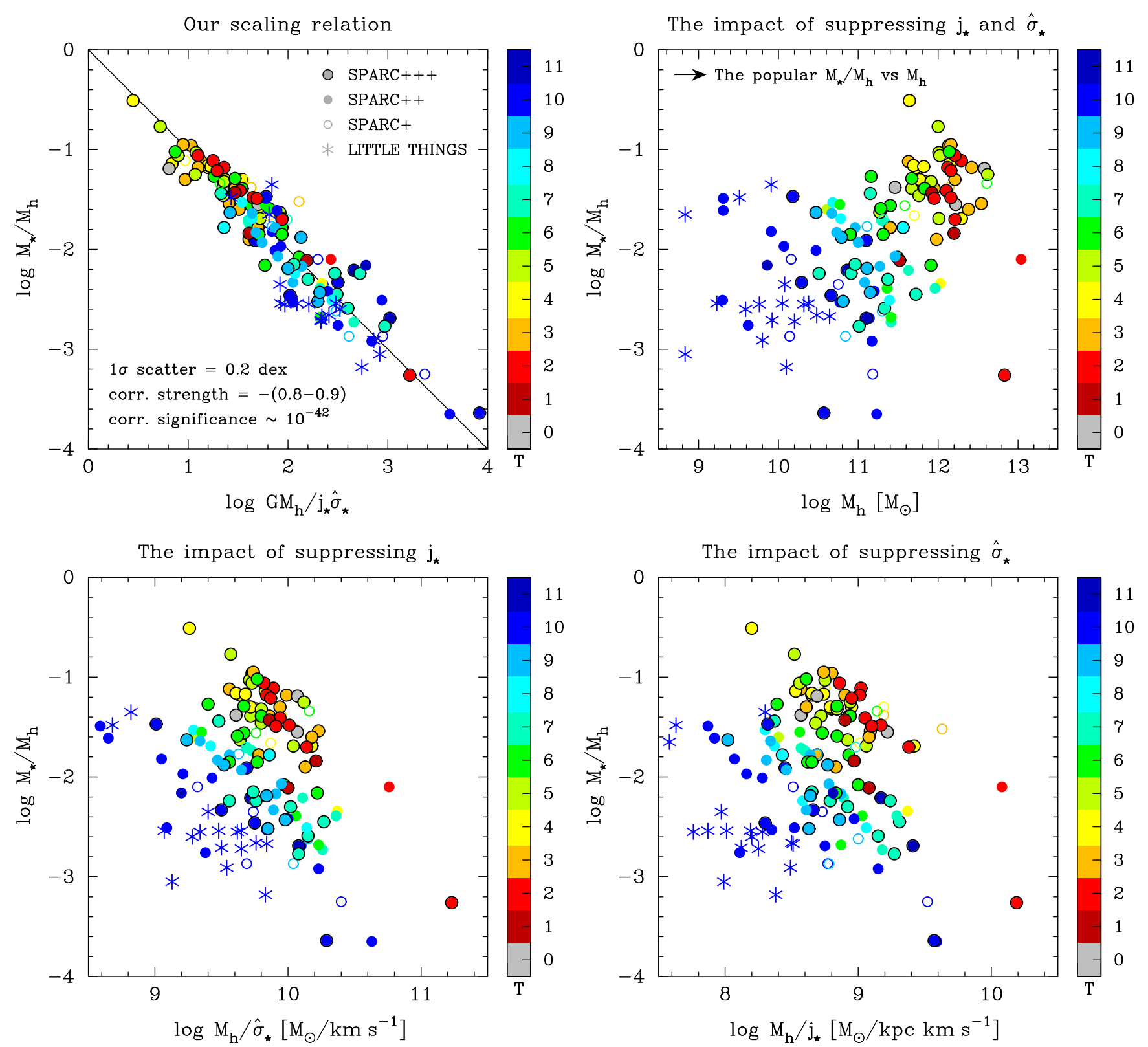

Figure 2. Our scaling relation, $M_{\star} / M_{\mathrm{h}}$ vs $G M_{\mathrm{h}} / j_{\star} \hat{\sigma}_{\star}$, and the impact of suppressing $j_{\star}$ and/or $\hat{\sigma}_{\star}$. Galaxies are colour-coded by Hubble stage, and galaxy samples are denoted as in Sect. 2. In the top-left panel, the diagonal line is the prediction based on disc gravitational instability, and statistical information about the data is given in summary form and simplified notation (see Sect. 2 for more information).

dispersion), and since mass is generally regarded as the most fundamental one, we ask ourselves: which of the other two quantities plays a more important role in our prediction? Fig. 2 answers this question. Suppressing $j_{\star}$ and/or $\hat{\sigma}_{\star}$ clearly has a strong impact on the result. In particular, suppressing both such quantities results in the popular $M_{\star} / M_{\mathrm{h}}$ vs $M_{\mathrm{h}}$ (see top-right panel), which is as poorly constrained as the relations shown in the bottom panels. Thus specific angular momentum and velocity dispersion play an equally important role in our prediction, and it is their interplay that constrains the SHMR so tightly in such a scenario.

\subsection{Residuals of the three SHMRs}

To fully characterize the three SHMRs, we have analysed their residuals, $\Delta \log \left(M_{\star} / M_{\mathrm{h}}\right)$, i.e. the deviations of the $\log \left(M_{\star} / M_{\mathrm{h}}\right)$ measurements from the underlying linear model (a line in a log-log plot). Remember that the model shown in the top-left panel of Fig. 2 is the prediction based on disc gravitational instability, while the models shown in the left and right panels of Fig. 1 are robust median-based fits to the data points (see figure caption for more information). Fits based on robust statistics like the median are especially useful when the data are few or contain a significant fraction of outliers, or even when the data deviate significantly from a normal distribution (see, e.g., Rousseeuw 1991; Press et al. 1992, chap. 15.7). 

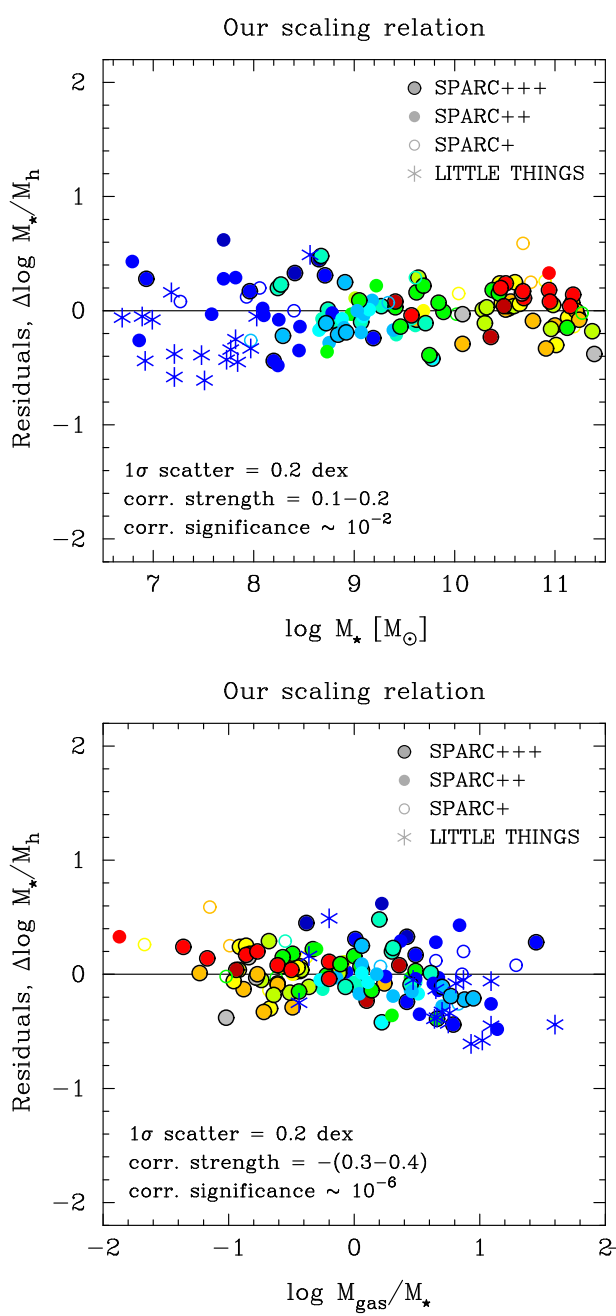

The popular $\mathrm{M}_{\star} / \mathrm{M}_{\mathrm{h}}$ vs $\mathrm{M}_{\mathrm{h}}$

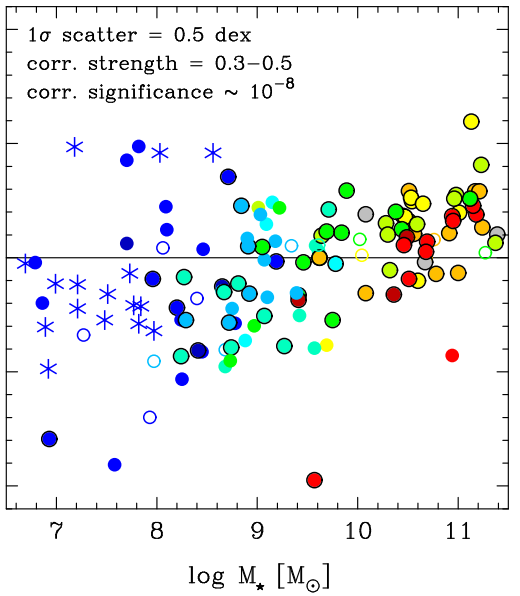

The popular $\mathrm{M}_{\star} / \mathrm{M}_{\mathrm{h}}$ vs $\mathrm{M}_{\mathrm{h}}$

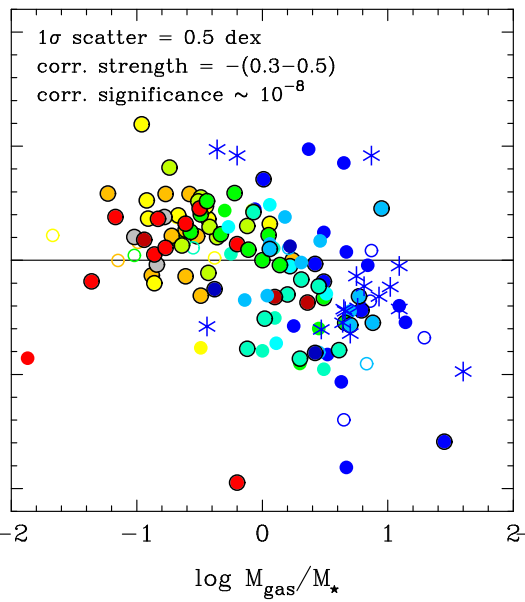

The popular $\mathrm{M}_{\star} / \mathrm{M}_{\mathrm{h}}$ vs $\mathrm{M}_{\star}$

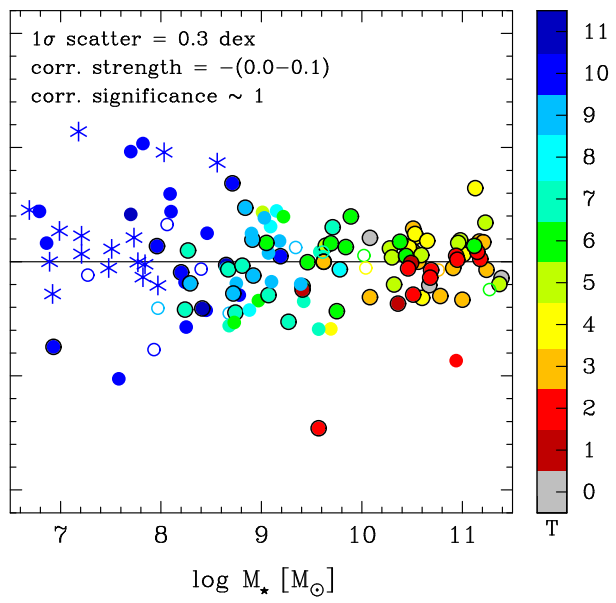

The popular $\mathrm{M}_{\star} / \mathrm{M}_{\mathrm{h}}$ vs $\mathrm{M}_{\star}$

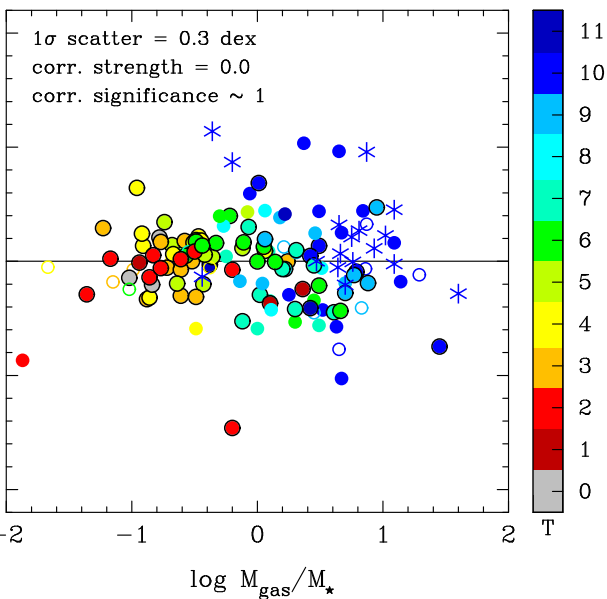

Figure 3. Residuals of the three SHMRs versus $M_{\star}$ (top panels) and $M_{\text {gas }} / M_{\star}$ (bottom panels). Galaxies are colour-coded by Hubble stage, and galaxy samples are denoted as in Sect. 2. Statistical information about the data is given in summary form and simplified notation (see Sect. 2 for more information).

The results of our analysis are illustrated in Fig. 3, both as a function of $M_{\star}$ (top panels) and as a function of $M_{\text {gas }} / M_{\star}$ (bottom panels). Our scaling relation shows a residual correlation with $M_{\text {gas }} / M_{\star}$, but this is a secondorder effect that depends on the accuracy of the measurements, and which vanishes in SPARC +++ . The two popular relations disclose an interesting difference: $M_{\star} / M_{\mathrm{h}}$ vs $M_{\star}$ shows no residual correlation at all, while $M_{\star} / M_{\mathrm{h}}$ vs $M_{\mathrm{h}}$ shows residual correlations with both $M_{\star}$ and $M_{\text {gas }} / M_{\star}$. This is true whether we consider the whole galaxy sample or SPARC +++ , and highlights two important facts. First, $M_{\star}$ is a more reliable estimator of $M_{\star} / M_{\mathrm{h}}$ than $M_{\mathrm{h}}$ : it is unbiased, and it also has less scatter. Second, the large scatter of $M_{\star} / M_{\mathrm{h}}$ vs $M_{\mathrm{h}}$ hides a weak residual trend with stellar mass and reverse trend with gas mass fraction, i.e. massive gas-poor galaxies (lenticulars and early-type spirals) tend to have a higher stellar-to-halo mass ratio than expected, and vice versa for low-mass gas-rich galaxies (late-type spirals and dwarfs).

\section{OUR DIAGNOSTICS AS A CRITICAL TEST FOR SIMULATIONS OF GALAXY FORMATION AND EVOLUTION}

The SHMR is not only one of the main sources of information we have on the galaxy-halo connection, but also an important indicator of the performance of galaxy formation models (e.g., Dutton et al. 2011; Read et al. 2017; Forbes et al. 2019; Agertz et al. 2020; Marasco et al. 2020, hereafter M20; Rodríguez-Puebla et al. 2020; Zanisi et al. 2020). Here we join this effort and demonstrate the importance of our results for the simulation community.

We focus on central, regularly rotating, massive $\left(M_{\star}>\right.$ $\left.5 \times 10^{10} \mathrm{M}_{\odot}\right)$ disc galaxies at redshift $z=0$, and compare the result from the SPARC dataset with the results from two recent cosmological hydrodynamical simulations: run Ref-L0100N1504 of EAGLE (Schaye et al. 2015), and run TNG100-1 of IllustrisTNG (Pillepich et al. 2018). We use the three galaxy samples analysed by M20, which were carefully selected so as to make such a comparison unbiased (see sect. 2 of M20). In particular, such galaxy samples cover a similar range of stellar masses $\left(M_{\star} \approx 10^{10.7-11.4} \mathrm{M}_{\odot}\right)$, hence they probe the same regime of the stellar mass function. 
Simulations vs Observations: Eq. (3)

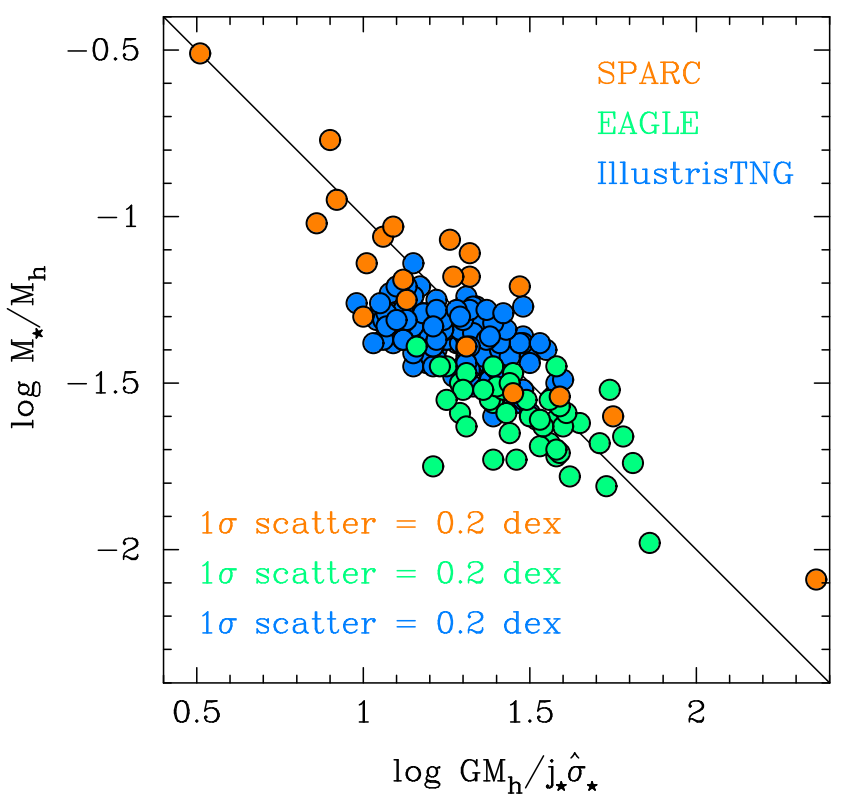

Simulations vs Observations: Eq. (1)

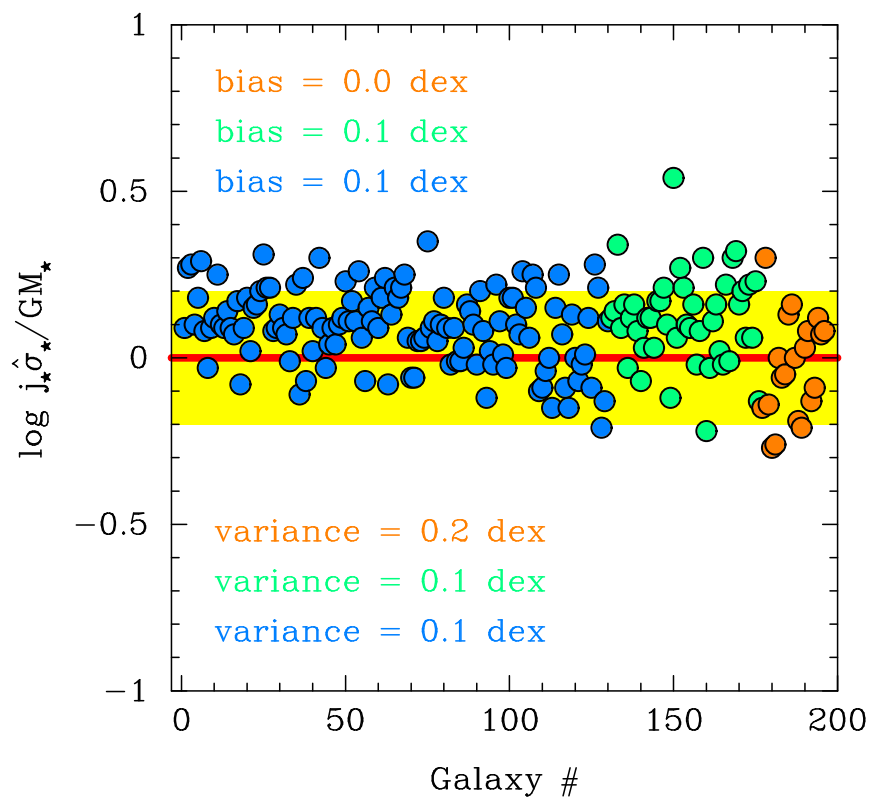

Figure 4. Our diagnostics as a critical test for simulations of galaxy formation and evolution: the case of central, regularly rotating, massive $\left(M_{\star}>5 \times 10^{10} \mathrm{M}_{\odot}\right)$ disc galaxies at redshift $z=0$. The lines in the left and right panels are the predictions based on disc gravitational instability. The shaded region in the right panel is the observed $1 \sigma$ scatter $(0.2$ dex for both Eqs 1 and 3$)$, measured considering the whole galaxy sample described in Sect. 2. More information is given in Sect. 4 .

M20 provided accurate measurements not only of $M_{\star}$ and $M_{\mathrm{h}}$ but also of $V_{\text {flat }}$, the circular speed at which the rotation curve flattens, and $R_{\text {eff }}$, the half-mass radius (see tables A.1 and A.2 of M20). This allows us to estimate the stellar specific angular momentum adopting a commonly used approximation: $j_{\star}=1.19 R_{\text {eff }} V_{\text {flat }}$ (e.g., Romanowsky \& Fall 2012). In reality, more accurate measurements of $j_{\star}$ are available for the SPARC sample (see Sect. 2), but we estimate $j_{\star}$ adopting the approximation above for consistency with the EAGLE and IllustrisTNG samples. Finally, we evaluate the velocity dispersion-based quantity $\hat{\sigma}_{\star}$ using Eq. (2) $[i=\star]$, as motivated in Sect. 3.2.

Fig. 4 provides compelling evidence that simulated and observed galaxies differ significantly in two complementary respects:

- The most striking result is the diverse distribution of galaxies along our scaling relation (see the left panel of Fig. 4). IllustrisTNG galaxies cluster around $M_{\star} / M_{\mathrm{h}}=4.3 \times$ $10^{-2}$, while EAGLE galaxies cluster around $M_{\star} / M_{\mathrm{h}}=2.5 \times$ $10^{-2}$. This is in sharp contrast to SPARC galaxies, which stretch over a range of two orders of magnitude and have median $M_{\star} / M_{\mathrm{h}}=6.6 \times 10^{-2}$. Thus such simulations not only underestimate the median of $M_{\star} / M_{\mathrm{h}}$, as pointed out by M20, but also dramatically misrepresent the large covariance of $M_{\star} / M_{\mathrm{h}}$ and $G M_{\mathrm{h}} / j_{\star} \hat{\sigma}_{\star}$.

- The other result concerns the scatter across our scaling relation (see again the left panel of Fig. 4). On the one hand, simulated galaxies have the same $1 \sigma$ scatter as observed ones, which is 0.2 dex regardless of whether we consider SPARC galaxies or the whole galaxy sample analysed in Sect. 3. On the other hand, simulated galaxies are systematically offset from our prediction (the diagonal line), hence their 'scatter' is partly variance and partly bias. To illustrate this result more eloquently, we make use of the key Eq. (1) $[i=\star]$ (see now the right panel of Fig. 4). IllustrisTNG and EAGLE galaxies show equal amounts of variance and bias, whereas SPARC galaxies show twice as much variance and no bias at all. Thus such simulations produce discs that are too gravitationally stable and have too little variance in $Q_{\star}$, the stellar Toomre parameter.

The mismatch in statistical properties between simulations and observations demonstrates the challenge of reproducing the efficiency of galaxy formation. This could be due to the inability of current cosmological-volume simulations to resolve the structure of the ISM, which is crucial for capturing not only the turbulent nature of star formation but also the subsequent coupling between feedback and (inter)galactic scales. Furthermore, resolving the fragmented nature of the gas affects how AGN fuelling proceeds. While these are challenges for present-day models of galaxy formation and evolution, they will be resolved by future generations of simulations, which aim to cover a wider dynamical range. However, the issue of choosing adequate sub-grid recipes will remain even with improved numerical resolution. Unless this problem gets eventually solved by implementations reaching the predictive level of first principles, sub-grid recipes will still be potentially important sources of discrepancy between simulated and observed statistical properties of galaxies, in particular their formation efficiency.

\section{CONCLUSIONS}

Using high-quality data for late-type galaxies of all morphological types (S0-BCD) from the SPARC and the LITTLE THINGS samples, we have performed a detailed compara- 
tive analysis of three scaling relations: two popular forms of the SHMR, $M_{\star} / M_{\mathrm{h}}$ vs $M_{\mathrm{h}}$ and $M_{\star} / M_{\mathrm{h}}$ vs $M_{\star}$, and a scaling relation driven by disc gravitational instability, $M_{\star} / M_{\mathrm{h}}$ vs $G M_{\mathrm{h}} / j_{\star} \hat{\sigma}_{\star}$, where $j_{\star}$ is the stellar specific angular momentum and $\hat{\sigma}_{\star}$ is the stellar radial velocity dispersion (properly averaged and rescaled). Our major conclusions are pointed out below.

- $M_{\star} / M_{\mathrm{h}}$ vs $M_{\mathrm{h}}$ has a large scatter $(0.5 \mathrm{dex})$, which hides weak residual trends with stellar mass and gas mass fraction. $M_{\star}$ is a more reliable estimator of $M_{\star} / M_{\mathrm{h}}$ than $M_{\mathrm{h}}$ : it is unbiased, and it also has less scatter $(0.3 \mathrm{dex})$. This is true regardless of whether we consider the whole galaxy sample or the galaxies with most accurate measurements of $M_{\star}$ and $M_{\mathrm{h}}$. Thus $M_{\star} / M_{\mathrm{h}}$ vs $M_{\mathrm{h}}$ and $M_{\star} / M_{\mathrm{h}}$ vs $M_{\star}$ are not just two alternative parametrizations of the same relation, but two significantly different relations.

- $M_{\star} / M_{\mathrm{h}}$ vs $G M_{\mathrm{h}} / j_{\star} \hat{\sigma}_{\star}$ has a small scatter $(0.2 \mathrm{dex})$, which hides a very weak residual trend with gas mass fraction. But this is a second-order effect that vanishes if we consider the galaxies with most accurate measurements of $M_{\star}, M_{\mathrm{h}}$ and $j_{\star}$. The tightness of such a relation is impressive (see the top-left panel of Fig. 2), all the more so because the predicted stellar mass fraction is not a best-fitting model but results from a parameter-free theoretical approach (Romeo 2020).

- The only difference between $M_{\star} / M_{\mathrm{h}}$ vs $G M_{\mathrm{h}} / j_{\star} \hat{\sigma}_{\star}$ and $M_{\star} / M_{\mathrm{h}}$ vs $M_{\mathrm{h}}$ is the extra factor $G / j_{\star} \hat{\sigma}_{\star}$. So, although our scaling relation is perhaps not a proper SHMR since it cannot predict the halo mass explicitely, its tightness tells us that specific angular momentum and velocity dispersion may play significant roles in the galaxy-halo connection. In this exploratory work, we have disentangled such roles and shown that they are equally important. In fact, it is the interplay between such fundamental galaxy properties that constrains the SHMR so tightly in our scenario.

- Our scaling relation and the key Eq. (1) provide a critical test for simulations of galaxy formation and evolution. In this paper, we have illustrated how to make use of such diagnostics, and applied them to two recent cosmological hydrodynamical simulations: EAGLE and IllustrisTNG. Our diagnostics demonstrate that such simulations fail to reproduce several statistical properties of the galaxy-halo connection (see Fig. 4), which ultimately demonstrates how challenging it is to reproduce the efficiency of galaxy formation (see Sect. 4).

Such results have an important astrophysical implication. Since our scaling relation originates from the low galaxy-to-galaxy variance of Toomre's (1964) $Q$ stability parameter, the tightness of such a relation implies that the process of disc gravitational instability is, on average, remarkably uniform across disc galaxies of all morphological types: from lenticulars to blue compact dwarfs. This is intriguing, as we discuss below.

On the one hand, we know that the stellar Toomre parameter, $Q_{\star}$, is a reliable disc instability diagnostic in stardominated galaxies like lenticulars and spirals (Romeo \& Fathi 2016; Romeo \& Mogotsi 2017; Marchuk 2018; Marchuk \& Sotnikova 2018), provided that the value of $Q_{\star}$ is properly corrected so as to include disc thickness effects (e.g., Vandervoort 1970; Romeo \& Falstad 2013), and properly interpreted considering the influence of non-axisymmetric pertur- bations (e.g., Lau \& Bertin 1978; Griv \& Gedalin 2012; see sect. 5.2 of Romeo \& Fathi 2015 for an updated discussion).

On the other hand, we know that $Q_{\star}$ is strongly coupled to $Q_{\text {gas }}$ in gas-rich galaxies (e.g., Lin \& Shu 1966; Romeo \& Falstad 2013), and that the value of $Q_{\text {gas }}$ is affected by complex phenomena such as gas turbulence (Romeo et al. 2010; Romeo \& Agertz 2014), gas dissipation (Elmegreen 2011), stellar feedback (Agertz et al. 2015), and other non-linear processes (Inoue et al. 2016). The impact of such phenomena becomes particularly strong in gas-dominated galaxies like dwarf irregulars and blue compact dwarfs (Renaud 2019), where even the concept of 'disc' is not well defined.

Thus it is really intriguing that all such complexity averages out in a statistical sense, and that galaxies of such diverse morphology line up along the same scaling relation driven by disc gravitational instability! This is true not only for the SHMR but also for the atomic gas-to-stellar mass relation (see fig. 4 of Romeo 2020), which demonstrates that the cosmic variance of $Q$ is 0.2 dex, a universal value for both stars and atomic gas.

The astrophysical implication discussed above is a fundamental aspect of galaxy formation and evolution that deserves to be probed further. It is not an easy task to disentangle how much dynamical processes such as star formation, galaxy minor mergers and satellite accretion have contributed to the present-day value of $M_{\star}$, hence $M_{\star} / M_{\mathrm{h}}$ : whatever happens during that evolution, disc gravitational instability regulates $j_{\star}$ and $\hat{\sigma}_{\star}$ for a given $M_{\star}$ so as to fulfil Eq. (1) $[i=\star], j_{\star}(t) \hat{\sigma}_{\star}(t) \approx G M_{\star}(t)$, thus erasing the memory of those processes. It is not an easy task, but it can be done by means of properly designed numerical simulations and/or semi-analytic modelling, which we challenge the astrophysical community to perform.

\section{ACKNOWLEDGEMENTS}

We are very grateful to Lorenzo Posti for help with the data, and to Robert Nau for useful discussions. We are also very grateful to an anonymous referee for insightful comments and suggestions, and for encouraging future work on the topic.

\section{DATA AVAILABILITY}

The data underlying this article will be shared on reasonable request to the corresponding author.

\section{REFERENCES}

Agertz O., Kravtsov A. V., 2016, ApJ, 824, 79

Agertz O., Romeo A. B., Grisdale K., 2015, MNRAS, 449, 2156

Agertz O. et al., 2020, MNRAS, 491, 1656

Athanassoula E., 2008, MNRAS, 390, L69

Behroozi P., Wechsler R. H., Hearin A. P., Conroy C., 2019, MNRAS, 488,3143

Behroozi P. S., Wechsler R. H., Conroy C., 2013, ApJ, 770, 57

Binney J., Tremaine S., 2008, Galactic Dynamics. Princeton University Press, Princeton

Burkert A., 1995, ApJ, 447, L25

Carlberg R. G., Sellwood J. A., 1985, ApJ, 292, 79 
Christodoulou D. M., Shlosman I., Tohline J. E., 1995, ApJ, 443, 551

Cimatti A., Fraternali F., Nipoti C., 2020, Introduction to Galaxy Formation and Evolution: From Primordial Gas to PresentDay Galaxies. Cambridge University Press, Cambridge

Conroy C., Wechsler R. H., Kravtsov A. V., 2006, ApJ, 647, 201

Dutton A. A., Conroy C., van den Bosch F. C., Prada F., More S., 2010, MNRAS, 407, 2

Dutton A. A. et al., 2011, MNRAS, 416, 322

Efstathiou G., Lake G., Negroponte J., 1982, MNRAS, 199, 1069

Elmegreen B. G., 2011, ApJ, 737, 10

Forbes J. C., Krumholz M. R., Speagle J. S., 2019, MNRAS, 487, 3581

Girelli G., Pozzetti L., Bolzonella M., Giocoli C., Marulli F., Baldi M., 2020, A\&A, 634, A135

Griv E., Gedalin M., 2012, MNRAS, 422, 600

Hohl F., 1971, ApJ, 168, 343

Hunter D. A., Elmegreen B. G., 2006, ApJS, 162, 49

Hunter D. A. et al., 2012, AJ, 144, 134

Ianjamasimanana R., de Blok W. J. G., Heald G. H., 2017, AJ, 153, 213

Inoue S., Dekel A., Mandelker N., Ceverino D., Bournaud F., Primack J., 2016, MNRAS, 456, 2052

Iorio G., Fraternali F., Nipoti C., Di Teodoro E., Read J. I., Battaglia G., 2017, MNRAS, 466, 4159

Klypin A., Prada F., 2009, ApJ, 690, 1488

Lapi A., Salucci P., Danese L., 2018, ApJ, 859, 2

Lau Y. Y., Bertin G., 1978, ApJ, 226, 508

Leauthaud A. et al., 2012, ApJ, 744, 159

Lelli F., McGaugh S. S., Schombert J. M., 2016, AJ, 152, 157

Lin C. C., Shu F. H., 1966, Proc. Natl. Acad. Sci. USA, 55, 229

Mandelbaum R., Seljak U., Kauffmann G., Hirata C. M., Brinkmann J., 2006, MNRAS, 368, 715

Marasco A., Posti L., Oman K., Famaey B., Cresci G., Fraternali F., 2020, A\&A, 640, A70 (M20)

Marchuk A. A., 2018, MNRAS, 476, 3591

Marchuk A. A., Sotnikova N. Y., 2017, MNRAS, 465, 4956

Marchuk A. A., Sotnikova N. Y., 2018, MNRAS, 475, 4891

Miller R. H., Prendergast K. H., Quirk W. J., 1970, ApJ, 161, 903

Mo H. J., Mao S., White S. D. M., 1998, MNRAS, 295, 319

Mogotsi K. M., Romeo A. B., 2019, MNRAS, 489, 3797

More S., van den Bosch F. C., Cacciato M., Skibba R., Mo H. J., Yang X., 2011, MNRAS, 410, 210

Moster B. P., Somerville R. S., Maulbetsch C., van den Bosch F. C., Macciò A. V., Naab T., Oser L., 2010, ApJ, 710, 903

Moster B. P., Naab T., White S. D. M., 2013, MNRAS, 428, 3121

Müller J. W., 2000, J. Res. Natl. Inst. Stand. Technol., 105, 551

Navarro J. F., Frenk C. S., White S. D. M., 1996, ApJ, 462, 563

Okamura T., Shimasaku K., Kawamata R., 2018, ApJ, 854, 22

Ostriker J. P., Peebles P. J. E., 1973, ApJ, 186, 467

Pillepich A. et al., 2018, MNRAS, 473, 4077

Posti L., Fraternali F., Di Teodoro E. M., Pezzulli G., 2018, A\&A, 612, L6 (P18)

Posti L., Fraternali F., Marasco A., 2019, A\&A, 626, A56 (P19)

Press W. H., Teukolsky S. A., Vetterling W. T., Flannery B. P., 1992, Numerical Recipes in Fortran: The Art of Scientific Computing. Cambridge University Press, Cambridge

Read J. I., Iorio G., Agertz O., Fraternali F., 2017, MNRAS, 467, 2019

Renaud F., 2019, in Bragaglia A., Davies M., Sills A., Vesperini E., eds, Proc. IAU Symp. 351, Star Clusters: From the Milky Way to the Early Universe. Cambridge Univ. Press, Cambridge, p. 40

Rodríguez-Puebla A., Avila-Reese V., Yang X., Foucaud S., Drory N., Jing Y. P., 2015, ApJ, 799, 130

Rodríguez-Puebla A., Calette A. R., Avila-Reese V., RodriguezGomez V., Huertas-Company M., 2020, PASA, 37, 24
Romanowsky A. J., Fall S. M., 2012, ApJS, 203, 17

Romeo A. B., 2020, MNRAS, 491, 4843

Romeo A. B., Agertz O., 2014, MNRAS, 442, 1230

Romeo A. B., Falstad N., 2013, MNRAS, 433, 1389

Romeo A. B., Fathi K., 2015, MNRAS, 451, 3107

Romeo A. B., Fathi K., 2016, MNRAS, 460, 2360

Romeo A. B., Mogotsi K. M., 2017, MNRAS, 469, 286

Romeo A. B., Mogotsi K. M., 2018, MNRAS, 480, L23

Romeo A. B., Burkert A., Agertz O., 2010, MNRAS, 407, 1223

Rousseeuw P. J., 1991, J. Chemometrics, 5, 1

Schaye J. et al., 2015, MNRAS, 446, 521

Sellwood J. A., 2016, ApJ, 819, 92

Sellwood J. A., Carlberg R. G., 1984, ApJ, 282, 61

Toomre A., 1964, ApJ, 139, 1217

Vale A., Ostriker J. P., 2004, MNRAS, 353, 189

Valencia-Enríquez D., Puerari I., Rodrigues I., 2019, AJ, 157, 175

Vandervoort P. O., 1970, ApJ, 161, 87

van Uitert E. et al., 2016, MNRAS, 459, 3251

Wechsler R. H., Tinker J. L., 2018, ARA\&A, 56, 435

Zanisi L. et al., 2020, MNRAS, 492, 1671

Zoldan A., De Lucia G., Xie L., Fontanot F., Hirschmann M., 2018, MNRAS, 481, 1376

This paper has been typeset from a $\mathrm{T}_{\mathrm{E}} \mathrm{X} / \mathrm{L} \mathrm{L}_{\mathrm{E}} \mathrm{X}$ file prepared by the author. 